\title{
A rare case of pelvic Castleman's disease mimicking
}

\section{an adnexal tumor}

\author{
Erbil Karaman ${ }^{1^{\star}}$, Çağrı Ateş ${ }^{1}$, Ali Kolusarı ${ }^{1}$, İsmet Alkış ${ }^{1}$, Hanım Güler Şahin ${ }^{1}$, Abdulaziz Gül ${ }^{1}$, \\ Feyza Demir ${ }^{2}$
}

${ }^{1}$ Van Yuzuncu Yil University, Faculty of Medicine, Department of Gynecology and Obstatrics

${ }^{1} V$ an Yuzuncu Yil University, Faculty of Medicine, Department of Pathology Van Turkey

\begin{abstract}
Castleman's disease (CD) is described as a nodal lympho-follicular hyperplasia and mostly seen in thoracic cavity. The occurrence in pelvic cavity is very rare. It is usually mimicking the pelvic adnexal solid-heterogenous tumoral mass. So the preoperative diagnosis is mostly difficult. We now present a case of pelvic CD in a woman who complaint from pelvic pain. The patient underwent laparotomy with suspicious of adnexal tumor. However, the diagnosis was made at histopathologic examination postoperatively. The surgery was curative in this case and no recurrence was observed in the patient.
\end{abstract}

Key Words: Castleman's disease, pelvic pain, adnexal tumor

\section{Introduction}

Castleman's disease (CD) is a rare disease which is defined as non-malignant proliferation of lymphofollicular tissue and mainly seen in mediastinum and head-neck origin. It can reach giant size which can mimick the tumoral process (1). Its etology is still unknown and few cases in pelvic origin have been reported in the literature. Castleman firstly defined the disease by two microscopic features, lymphoid follicular hyperplasia and proliferation of microvascular structures with endothelial hyperplasia. Clinically, CD can be seen as two forms of unicentric CD (UCD) or multicentric with more systemic B-like symptoms. On literature search it was found that the most common locations of $\mathrm{CD}$ are the mediastinum $(63 \%)$, abdominal cavity $(11 \%)$, retroperitoneal region $(7 \%)$, axilla $(4 \%)$, and perirenal space $(2 \%)$ (2). Although the mediastinal involvement is the most common form, the genitourinary localization is highly infrequent and sometimes it creates great challenges during diagnosis and treatment management (3). The main issue in pelvic $C D$ is its mimicking capacity with regard to pelvic adnexial tumor either benign or malignant. And also, it is usually diagnosed postoperatively by histopathologic examination due to its rare occurrence and localization in pelvic region (4). So, with our case report, we want to report a woman with pelvic $\mathrm{CD}$ mimicking an adnexial tumor diagnosed postoperatively and to present its successful management with diagnostic imaging tools.

\section{Case Report}

A 52 years old perimenopousal patient came to our outpatient clinic with complaint of pelvic pain on left lower quadrant. On ultrasound image, it was seen as a $5 \times 5 \mathrm{~cm}$ solid adnexal mass similar like leiomyoma uteri. Then, MRI examination was performed and showed $4.8 \times 5.4 \mathrm{~cm}$ solid mass on left adnexa, situated just in pelvic region over left external iliac artery (figure 1 and 2). Parasitic myoma or a fibrotecoma originated from left ovary was suspected in preoperative assessment. The tumor markers including ca-125 and ca-19-9 were found to be normal. The patient underwent laparotomy and an enlarged uterus with adenomyosis with bilateral normal ovaries was found. Hysterectomy and bilateral oophorectomy performed. On exploration, a solid mass over the left external iliac artery covered by peritoneum was seen with the size of $5 \times 6 \mathrm{~cm}$. While the mass was excised, external iliac artery injury occured and repaired with primary suturing. The pathologic review showed that plasma type variant of Castleman's disease (figure 3). The patient was discharged uneventfully at 5 th postoperative day. 


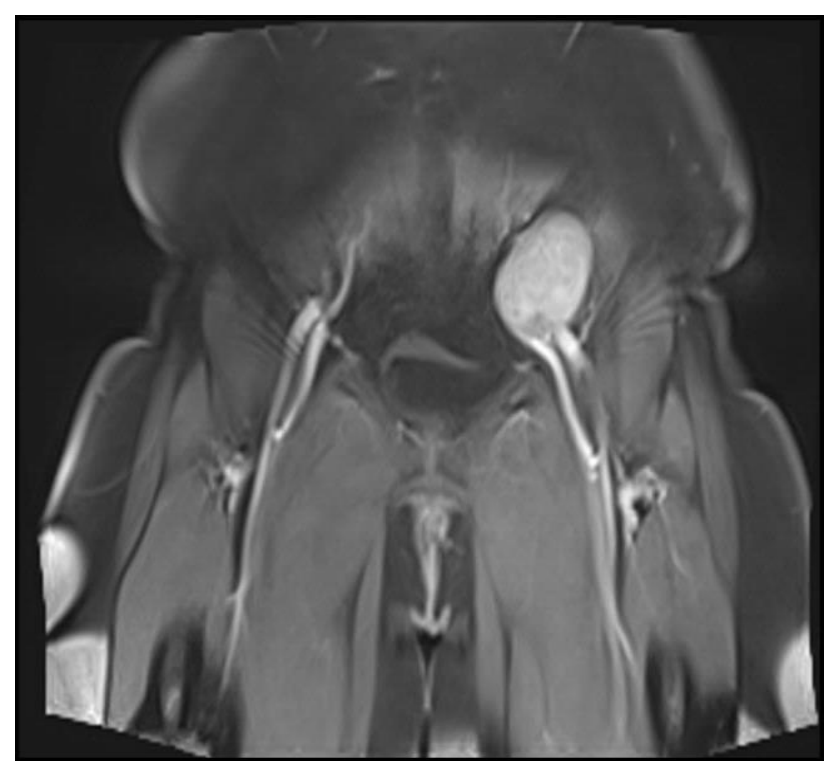

Fig. 1. Shows the solitary image in left adnexal region on coronal section of MRI examination

On follow-up examination, patient was sent to hematology clinic for detailed evaluation and treatment if needed. The patient was accepted as localized unicentric Castleman's disease with no systemic involvement and sign. So she did not receive any further treatment and the follow-up is going on currently.

\section{Discussion}

Castleman's disease is a complex group of lymphoproliferative disorders and most frequently localized in thoracic cavity. It is called as "a great mimic" in the literature because of it is generally thought as a tumoral mass in the different regions in the body. Castleman's Disease (CD) is known as a unique clinico-histopathological disease initially defined by Dr. Benjamin Castleman in 1956 (3). Castleman's article presented 13 patients with a clinical situation specified with benign lymphadenopathy seen in mediastinum.

Two histopathological variants of CD have been described - the hyaline vascular type and plasma cell type. The hyaline type shows significant hyperplasia of small hyalinized follicles with especially angioplastic follicular proliferation, on the other hand the plasma type has proliferative germinal centers, arrangements of plasma cells in the interfollicular area, increased in the presence vascular microstructures, and marked sinuses. It has been reported that $90 \%$ of $\mathrm{CD}$ belongs to the hyaline vascular variant. The majority of the hyaline vascular types have no symptom and are usually detected in routine chest $\mathrm{X}$-ray assessments. This type of tumor is generally in solid component, and operative excision

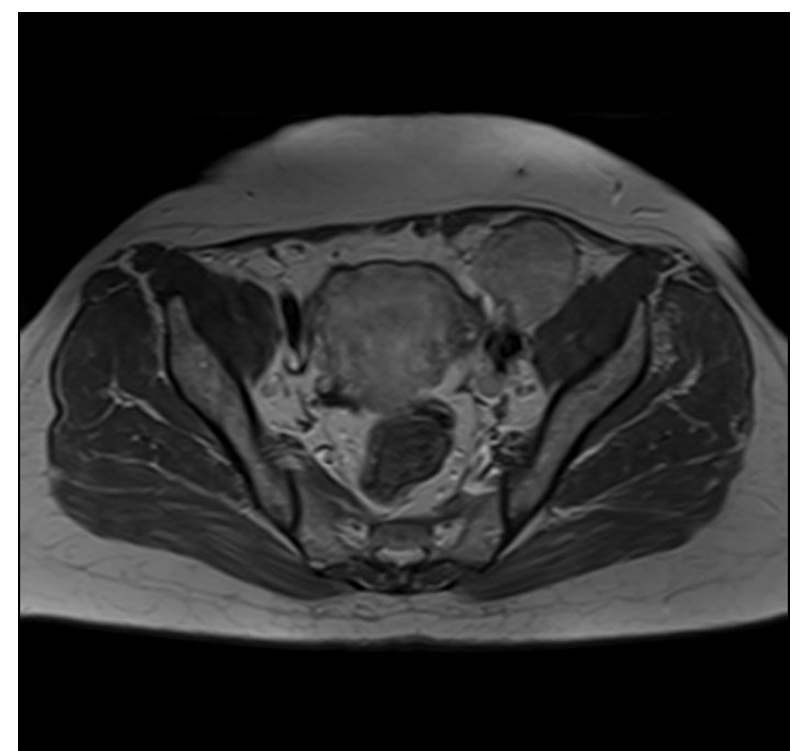

Fig. 2. Shows the solid mass seen on Pelvic MRI

almost always being a definitive treatment option. Pelvic CD generally involves this histopathological variant. However, in our case, the plasma cell variant discovered in pelvic cavity as a unicentric CD.

The plasma cell variant is sometimes multicentric and usually is diagnosed by multisystemic presentations such as fatigue, low red blood cell count, elevated gammaglobulin levels and a low thrombocyte level. This type of Castleman disease is sometimes associated with an immune deficiency condition and in some patients forwarded to malignant lymphoma (4). In our case, the CD was restricted to the left adnexial region with no systemic involvement or any laboratory manifestations.

In literature search, we found several case reports regarding pelvic Castleman's disease mimicking pelvic tumoral occurrence. Yelinen et al described a pelvic $\mathrm{CD}$ masquerading as a adnexal tumor. They stated that the tumor has some challenges regarding the diagnosis due to its solitary component, location and mobility (5). They could treat the patient with a second laparotomy due to missing the tumor during the first surgery. Their histopathology was hyalinvascular type of CD. In another case report by Ahmet et al reported a 24 year-old woman with complaint of urinary system and pelvic pain. In their case, the patient had systemic laboratory manifestations like preoperatively low plasma albumin, high thrombocyte level and negative albumin globulin ratio. The patient's systemic findings resolved postoperatively and they concluded that the diagnosis of this entity is difficult even by the frozen section intraoperatively (6).

In our case, the patient had no systemic symptom and the tumoral mass was thought as an adnexal tumoral 


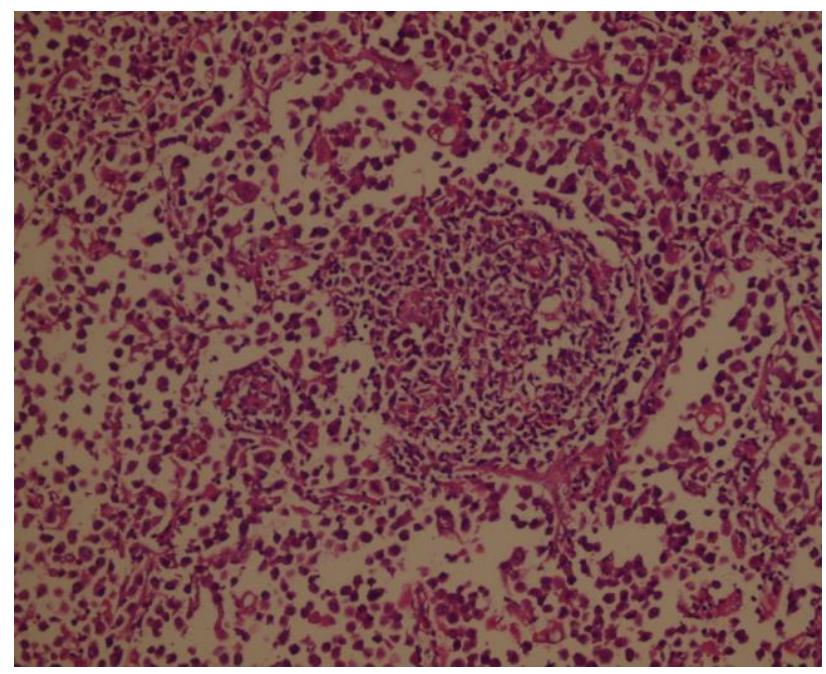

Fig. 3. Shows the histopathological picture of Castleman's disease, plasma cell variant with presence of marked plasma cells around follicles with prominence of germinal centers

occurrence preoperatively. The MRI examination could not distinguish this disease from a solitary adnexal tumor. In conclusion, the possibility of Castleman's disease should be taken into account in mind when considering a pelvic mass. Castleman's disease should be included within the differential diagnosis of pelvic masses on evaluation with pelvic imaging studies like MRI.

\section{References}

1. Keller AR, Hochholzer L, Castleman B. Hyalinevascular and plasma-cell types of giant lymph node hyperplasia of the mediastinum and other locations. Cancer 1972; 29: 670-683.

2. Riba PO. Castleman's disease. Angiofollicular benign lymph-node hyperplasia. Radiological features in differential diagnosis of vascular tumor in the pelvis. Br J Radiol 1979; 52: 412-414.

3. Castleman B, Iverson I, Menendez VP. Localized mediastinal lymph-node hyperplasia resembling thymoma. Cancer 1956; 9: 822-830.

4. Hsieh $\mathrm{CH}$, Changchien CC, Lan KC, et al. Pelvic Castleman's disease presenting as an adnexal tumor. Acta Obstet Gynecol Scand 2004; 83: 311313.

5. Yelinen K, Sarlomo-Rikala M, Laatikainen T. Pelvic Castleman disease mimicking adnexal tumor. Obstet Gynecol 1995; 85: 894-897.

6. Ahmet ZI, Aydemir E, Zorlu G, et al. Pelvic Castleman disease: an unusual pelvic mass. Aust N Z J Obstet Gynecol 1994; 34: 118-120. 